\title{
Role of comorbidities in a cohort of patients with COPD undergoing pulmonary rehabilitation
}

\author{
E Crisafulli, ${ }^{1}$ S Costi, ${ }^{2}$ F Luppi, ${ }^{2}$ G Cirelli, ${ }^{1}$ C Cilione, ${ }^{1}$ O Coletti, ${ }^{1}$ L M Fabbri, ${ }^{2}$ E M Clinii, ${ }^{1}$
}

- Supplementary statistical data are published online only at http://thorax.bmj.com/content/ vol63/issue6

${ }^{1}$ Ospedale Villa Pineta, Pavullo (Modena), Modena, Italy;

${ }^{2}$ Section of Respiratory

Diseases, Department of Oncology, Haematology and

Pneumology, University of

Modena-Reggio Emilia, Modena, Italy

Correspondence to:

Professor E M Clini, University of

Modena and Ospedale Villa

Pineta, Department of Oncology, Haematology and Pneumology,

Ospedale Villa Pineta, Via Gaiato 127, 41026 Pavullo (MO), Italy; enrico.clini@unimore.it

Received 28 June 2007 Accepted 4 December 2007

Published Online First

18 January 2008

\section{ABSTRACT}

Background: Chronic obstructive pulmonary disease (COPD) is often associated with other chronic diseases. These patients are often admitted to hospital based rehabilitation programmes.

Objectives: To determine the prevalence of chronic comorbidities in patients with COPD undergoing pulmonary rehabilitation and to assess their influence on outcome.

Design: Observational retrospective cohort study. Setting: A single rehabilitation centre.

Patients: 2962 inpatients and outpatients with COPD (73\% male, aged 71 (SD 8) years, forced expiratory volume in $1 \mathrm{~s}\left(\mathrm{FEV}_{1}\right) 49.3$ (SD 14.8)\% of predicted), graded 0,1 or $\geqslant 2$ according to the comorbidity categories and included in a pulmonary rehabilitation programme.

Measurements: The authors analysed the number of self-reported comorbidities and recorded the Charlson Index. They then calculated the percentage of patients with a predefined positive response to pulmonary rehabilitation (minimum clinically important difference (MCID)), as measured by improvement in exercise tolerance (6 min walking distance test (6MWD)), dyspnoea (Medical Research Council scale) and/or health related quality of life (St George's Respiratory Questionnaire (SGRQ)).

Results: $51 \%$ of the patients reported at least one chronic comorbidity added to COPD. Metabolic (systemic hypertension, diabetes and/or dyslipidaemia) and heart diseases (chronic heart failure and/or coronary heart disease) were the most frequently reported comorbid combinations (61\% and $24 \%$, respectively) among the overall diseases associated with COPD. The prevalence of patients with MCID was different across the comorbidity categories and outcomes. In a multiple categorical logistic regression model, the Charlson Index (OR $0.72(96 \% \mathrm{Cl}$ 0.54 to 0.98 ) and 0.51 (96\% $\mathrm{Cl} 0.38$ to 0.68 ) vs $6 \mathrm{MWD}$ and SGRO, respectively), metabolic diseases (OR 0.57 ( $96 \% \mathrm{Cl} 0.49$ to 0.67 ) vs $6 \mathrm{MWD}$ ) and heart diseases (OR 0.67 (96\% CI 0.55 to 0.83 ) vs SGRO) reduced the probability to improve outcomes of rehabilitation.

Conclusions: Most patients with COPD undergoing pulmonary rehabilitation have one or more comorbidities. Despite the fact that the presence of comorbidities does not preclude access to rehabilitation, the improvement in exercise tolerance and quality of life after rehabilitation may be reduced depending on the comorbidity.

Chronic obstructive pulmonary disease (COPD) is one of the leading causes of morbidity and mortality. ${ }^{12}$ Although alarming, this probably underestimates the true burden of this disease on health status, health care costs and overall actual and projected prognoses. ${ }^{3}$ COPD is also an important risk factor for other chronic diseases that contribute to morbidity and mortality. ${ }^{4}$

Comorbidities are defined as other chronic medical conditions that accompany a disease process, ${ }^{5}$ and they are particularly prevalent in the elderly. ${ }^{6-8}$ The most common chronic conditions associated with COPD are hypertension, diabetes, coronary artery disease, heart failure, pulmonary infections, cancer and pulmonary vascular disease. ${ }^{9-14}$ Comorbidities are an important determinant of health related quality of life in patients with COPD. ${ }^{13} 1516$

Pulmonary rehabilitation is a non-pharmacological comprehensive intervention, effective in the long term management of symptomatic COPD of all grades of severity, ${ }^{17}$ even in the elderly. ${ }^{18}$ Pulmonary rehabilitation improves symptoms, quality of life and exercise performance, ${ }^{19}$ and is effective in decreasing consumption of health care resources. ${ }^{20}$

The aim of this study was to determine the frequency and prevalence of chronic comorbidities in patients with COPD and to assess their influence on the effects of pulmonary rehabilitation.

\section{METHODS \\ Patients}

Our study was approved by the our institutional review board.

Of all the patients who were admitted to hospital for rehabilitation purposes from January 2003 to December $2005(n=4055)$, we selected 2962 patients with COPD for retrospective analysis. Patients were selected according to the following criteria: primary diagnosis of (i) COPD (491 codes), as defined and classified according to the Global Initiative for Chronic Obstructive Lung Disease (GOLD) guidelines ${ }^{19}$ and/or (ii) pulmonary emphysema (492 code) and (iii) respiratory failure (518.8 $\times$ codes). Spirometric severity of COPD was established according to the GOLD guidelines. ${ }^{21}$ Therefore, patients with asthma or any other pulmonary diseases (either obstructive or restrictive) were excluded from the study. Discharge diagnosis was made by a physician specialising in pulmonary medicine. The diagnosis of each patient was recorded on an electronic database according to the codes of the International Classification of Diseases, version 9-CM. ${ }^{22}$

\section{Comorbidities}

Comorbid conditions were diagnosed according to the International Classification of Health Problems in Primary $\mathrm{Care}^{23}$ and retrieved from the medical files. All patients were grouped according to the following comorbidity categories: 0 (absence of 
Table 2 Patients with a predefined positive response (MCID) to pulmonary rehabilitation in terms of improvement in exercise tolerance, dyspnoea and quality of life

\begin{tabular}{|c|c|c|c|c|c|}
\hline & \multirow{2}{*}{$\begin{array}{l}\text { All patients } \\
\text { ( } \mathrm{n}=2962)\end{array}$} & \multicolumn{3}{|c|}{ Comorbidity categories } & \multirow[b]{2}{*}{$\chi^{2}$} \\
\hline & & $0(n=1443)$ & $1(n=1136)$ & $\geqslant 2(n=383)$ & \\
\hline 6MWD +54 m (n (\%)) & $1822(61.5)$ & $904(62.6)$ & $680(59.8)$ & $238(62.4)$ & 0.298 \\
\hline MRC grade -1 (n (\%)) & $2416(81.5)$ & $1170(81.0)$ & $914(80.3)$ & 332 (87.1) & 0.010 \\
\hline SGRO total $-4(\mathrm{n}(\%))$ & $1845(62.2)$ & $962(66.6)$ & $658(57.9)$ & $225(59.0)$ & 0.001 \\
\hline Withdrawal rate (\%) & 2.82 & 1.89 & 3.44 & 1.26 & 0.561 \\
\hline
\end{tabular}

Data are presented as mean (SD).

GMWD, 6 min walking distance test; MCID, minimum clinically important difference; MRC, Medical Research Council dyspnoea score; SGRQ, St. George's Respiratory Questionnaire total score.

shown in fig $1 \mathrm{~B}$. Heart disease (24\%), metabolic disease (62\%) and skeletal disease $(7 \%)$ were the most frequently reported.

Taking the whole cohort of COPD into account, systemic arterial hypertension was the most prevalent comorbidity $(27.4 \%)$, followed by chronic heart failure $(10.5 \%)$ and diabetes $(10.3 \%)$, whereas the prevalence of combined heart disease, metabolic disease and skeletal disease was 16\%, 38\% and 5\%, respectively (figure not displayed).

Mean changes after pulmonary rehabilitation were 67 (SD 47) $\mathrm{m}$ in the $6 \mathrm{MWD},-1.1$ (SD 0.7) for the MRC score and -6.4 (SD 4.5) for the SGRQ score. However, the number and per cent of patients with MCID in the MRC score and SGRQ score (but not in the 6MWD) were different $(p<0.05)$ across the comorbidity categories (table 2). Withdrawal rate from pulmonary rehabilitation (3\%) was no different across the same categories (table 2).

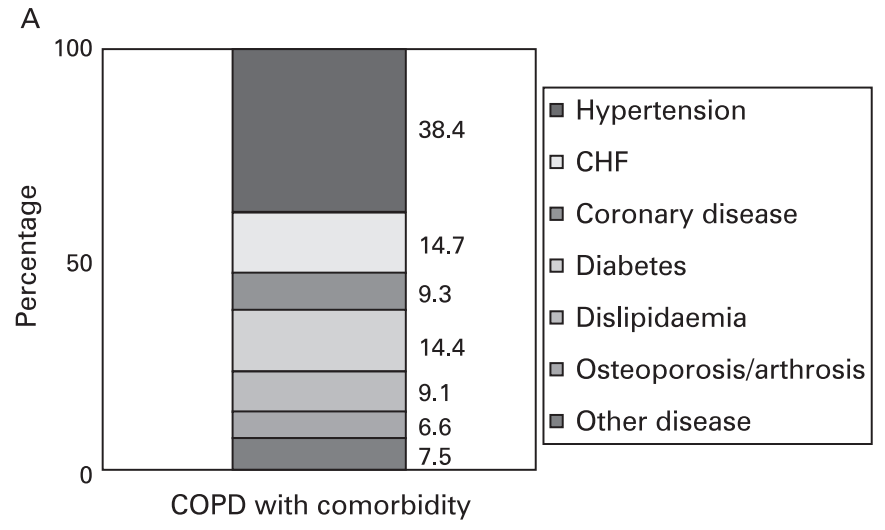

B

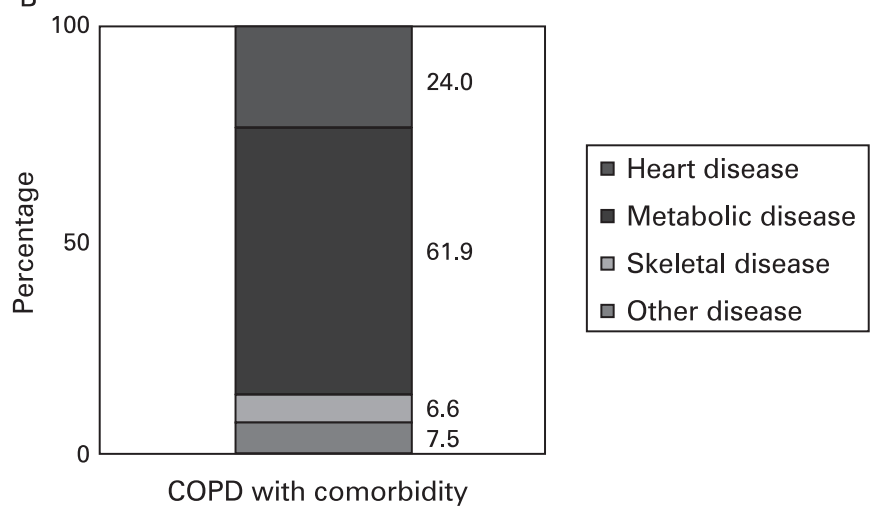

Figure 1 Frequency distribution (\% of total) of individual chronic comorbidities (A) and combinations of comorbidities (B) added to chronic obstructive pulmonary disease (COPD) in the population. CHF, chronic heart failure.
Univariate analysis for binary variables showed that age (with SGRQ, $p=0.001$ ), Charlson Index (with 6MWD and SGRO, $\mathrm{p}=0.001$ ), forced expiratory volume in $1 \mathrm{~s}\left(\mathrm{FEV}_{1}\right)$ (with MRC, $p=0.032$ ), heart disease (with 6MWD and SGRQ, $p=0.001$ ), metabolic disease (with 6MWD, $p=0.001$; MRC, $p=0.004$; SGRQ, $p=0.043$ ) and skeletal disease (with MRC, $p=0.049$ ) were significantly related to outcome and were then entered into the multivariate analysis (for detailed statistics, see also the table in the online supplement material).

Table 3 displays the results of the multiple logistic regression analysis. Age, $\mathrm{FEV}_{1}$, Charlson Index, and heart disease and metabolic disease combinations entered the prediction equation as independent variables. In particular, airway obstruction significantly predicted the improvement in MRC score; Charlson Index and metabolic disease were inversely related to improvement in $6 \mathrm{MWD}$; and heart disease directly and indirectly predicted the improvement in $6 \mathrm{MWD}$ and SGRO, respectively.

The overall results did not change when taking the subgroups of patients with COPD undergoing inpatient and outpatient (13\% of total) rehabilitation.

\section{DISCUSSION}

Our study showed that most patients with COPD undergoing pulmonary rehabilitation have one or more chronic comorbidities and that the severity of comorbidities, particularly the simultaneous presence of metabolic and/or heart diseases, significantly reduces the beneficial effects of pulmonary rehabilitation on exercise tolerance and perceived quality of life.

Complex chronic comorbidities may significantly affect the clinical severity of COPD, ${ }^{71} 18$ being present in up to $56 \%$ of patients with COPD compared with non-COPD subjects of the same age. ${ }^{32}$ The prevalence of chronic comorbidities varies among studies. ${ }^{95}$ Although the methodology was similar, the prevalence of at least one comorbidity in our cohort was lower (approximately 65\%) than the prevalence reported in other studies $(>70 \%){ }^{34}{ }^{35}$ Indeed, the population we examined was selected because it included only patients referred for pulmonary rehabilitation. The prevalence of comorbidities in patients with COPD referred for pulmonary rehabilitation has not been definitively examined previously ${ }^{36}{ }^{37}$ probably because of the relatively low number of patients and inclusion/exclusion criteria of pulmonary rehabilitation. Most $(>90 \%)$ patients included in our study had moderate to severe COPD (stages 2 and 3 according to the GOLD guidelines), suggesting that patients with very severe (stage 4) COPD, possibly with more and more severe chronic comorbidities, ${ }^{68}$ are less frequently referred for rehabilitation. Interestingly, the reported comorbidity Charlson score ${ }^{24} 39$ in our patients was similar $(1.26-2.85)$ to that reported in other studies. ${ }^{36} 4041$ 
Table 3 Factors predicting pulmonary rehabilitation outcome in the cohort of patients with COPD

\begin{tabular}{llrllll}
\hline Dependent variable & Variable & $\boldsymbol{\beta}$ & $\mathbf{S E}$ & $\mathbf{E x p}(\boldsymbol{\beta}) \mathbf{O R}$ & $\mathbf{9 6 \%}$ CI & $\mathbf{p}$ Value \\
\hline 6MWD improvement & Charlson Index & -0.31 & 0.14 & 0.72 & 0.54 to 0.98 & 0.024 \\
& Heart disease & 0.86 & 0.11 & 2.36 & 1.85 to 3.01 & 0.001 \\
& Metabolic disease & -0.55 & 0.07 & 0.57 & 0.49 to 0.67 & 0.001 \\
MRC improvement & FEV & 0.53 & 0.13 & 1.71 & 1.34 to 2.40 & 0.001 \\
& Metabolic disease & 0.16 & 0.09 & 1.17 & 0.93 to 1.77 & 0.104 \\
& Skeletal disease & -0.36 & 0.20 & 0.69 & 0.66 to 1.48 & 0.074 \\
SGRQ Improvement & Age & 0.30 & 0.07 & 1.35 & 1.15 to 1.59 & 0.001 \\
& Charlson Index & -0.66 & 0.14 & 0.51 & 0.38 to 0.68 & 0.001 \\
& Heart disease & -0.38 & 0.10 & 0.67 & 0.55 to 0.83 & 0.001 \\
& Metabolic disease & -0.09 & 0.07 & 0.91 & 0.77 to 1.07 & 0.249 \\
\hline
\end{tabular}

For details of the included variables, see the results section.

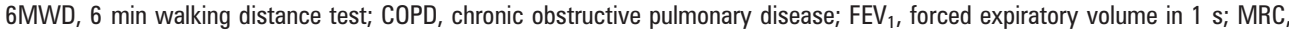
Medical Research Council dyspnoea score; SGRQ, St George's Respiratory Questionnaire total score.

Systemic hypertension, chronic heart failure, diabetes, coronary heart disease, dyslipidaemia and osteoporosis-arthrosis ranked as the six most frequent among all reported comorbidities; these six represented $>85 \%$ altogether (fig 1A), and their proportions were similar to those reported in previous studies. ${ }^{37}$ The same features were also observed with regard to the prevalence of these diseases within the cohort of studied COPD (see also results).

Metabolic disease and heart disease were the two most frequent disease combinations among all reported comorbidities (fig 1B). It is noteworthy that the term metabolic disease only approximates the internationally shared definition of "metabolic syndrome". ${ }^{42}$ Notwithstanding, metabolic alterations together with chronic heart diseases are well known to independently worsen the prognosis of adults. ${ }^{1-3}$ In elderly patients with complex comorbidities, obesity and low physical activity are factors that increase the risk of death by $15 \%$ and $3 \%$, respectively. ${ }^{3}$ Both of these factors are important determinants of survival in patients with $\mathrm{COPD}^{36} 43^{44}$ and are likely to be improved with comprehensive rehabilitation. ${ }^{17}$ Therefore, it is not surprising to find this frequent pattern of comorbidities in patients with COPD enrolled in pulmonary rehabilitation programmes.

Exercise performance, dyspnoea and quality of life (as assessed by the SGRQ) are widely recognised as important patient centred outcomes of COPD, whose favourable change indicates the clinical effect of treatment, ${ }^{21}$ particularly pulmonary rehabilitation. ${ }^{45}$ The MCID method adopted here to define the a priori criteria for outcome improvement seems appropriate in this field. ${ }^{29}$ The proportion of patients with significant changes in MRC (higher percentage in category $\geqslant 2$ ) and SGRO (higher percentage in category 0) was different across the comorbidity categories (table 2). However, the magnitude of their change after rehabilitation was similar in these groups, confirming the ability of pulmonary rehabilitation to individually target (whenever possible) the reasonably reachable goals for reducing disability.

The proportion of patients who reported a positive change in all three pulmonary rehabilitation outcomes in our study was $>50 \%$. Overall, this percentage was higher than that in a previous study ${ }^{29}$ in outpatients with COPD. These differences may be partially explained by the different site (inpatient facility for most of our patients with COPD) and grade of physical disability (mean 285 vs $335 \mathrm{~m}$ for the 6MWD in De Torres and colleagues ${ }^{29}$ and in our study, respectively) recorded at baseline.

To our knowledge, our study is the first to investigate the predictive role of comorbidities on the effect of pulmonary rehabilitation. To date, this information is still lacking in the field of rehabilitation with regard to patients with COPD. Only one study ${ }^{38}$ has considered the independent role of comorbidities on functional outcomes (balance and gait) after physical intervention in over 700 elderly patients who were recovering from stroke or who had Parkinson's disease or osteoarthritis. The investigators observed that the determinants of poor recovery were characterised by the combination of "more disabling diseases" (COPD, heart failure, peripheral artery disease, diabetes and cancer) rather than the effect of each chronic disease, independent of age, cognitive status or functional status at admission.

Our findings are consistent with those of De Fazio and colleagues $^{38}$; indeed, we have shown that the combinations of chronic comorbidities (metabolic and/or heart diseases), but not each chronic disease per se associated with COPD, independently predict improvement after pulmonary rehabilitation (table 3). In addition, the overall impact of comorbidities (here quantified by means of the Charlson Index) inversely predicts the improvement of both exercise tolerance and quality of life after rehabilitation. This suggests that the more complex cases are those less likely to benefit from pulmonary rehabilitation.

The presence of associated metabolic disorders is inversely related to improvement in $6 \mathrm{MWD}$. Indeed, it is likely that the systemic complications associated with hypertension, diabetes, dyslipidaemia and overweight limit the ability to improve physical performance in terms of exercise tolerance, as known in humans. ${ }^{46}$

Interestingly, the presence of combined heart diseases acts as both positive and negative predictors depending on the outcome investigated. In particular, it directly relates to the improvement in $6 \mathrm{MWD}$ but inversely relates to the change in quality of life (SGRO).

Despite the large contribution that cardiac dysfunction may give per se to health status and prognosis in adults, ${ }^{47}$ training during rehabilitation in these chronic diseases appears to be recommended. ${ }^{48}$ These diseases, even if associated with COPD, are not likely to alter the individual's ability to improve his or her physical performance. On the other hand, the combination with chronic heart diseases (but not of the metabolic disorders) inversely predicts the effect of pulmonary rehabilitation on the quality of life of patients with COPD. We can only speculate that this particular interaction and disease complexity may negatively impact on the potential benefit of rehabilitation on the individual's perceived health status, as reported previously. ${ }^{49}$

Thus on the one hand the presence of more complex comorbidities among patients with COPD undergoing 
pulmonary rehabilitation does not necessarily mean that this process is likely to benefit those patients less. On the other hand, however, it probably implies that outcomes should be better targeted and intervention should be better tailored to take account of this factor. Interestingly, despite the fact that rehabilitation in our cohort of COPD was delivered in two different setting (13\% as outpatients), the overall results were similar in these subgroups of patients, confirming a quite homogeneous cohort and making the clinical message even stronger.

Despite our new and original findings, our study has limitations which need to be addressed. Firstly, this is a retrospective cohort analysis and hence a further prospective trial is needed to confirm the results. Secondly, the pattern of comorbidities in our single centre COPD cohort, although consistent, should be confirmed in a multicentre study where objective confirmation on the single diagnosis is also considered. Thirdly, diagnosis of the comorbidities was based on a selfreported method; therefore, the predictive role of the metabolic and heart diseases on the effects of pulmonary rehabilitation need to be corroborated by more precise biological or pathogenetic indicators. Fourthly, other potential individual factors such as socioeconomic status, smoking habit and pack years were not retrospectively available nor were they taken into account as potential confounders in the predictive analysis.

Notwithstanding these important factors which limit the generalisability of this study by using a single site, our findings underline the necessity to globally assess patients with COPD for their functions and comorbidities in order to determine the most appropriate approach for treating them. ${ }^{50}$

In conclusion, this study shows that chronic comorbidities are very frequent in patients with COPD undergoing rehabilitation but a positive effect is reached in $>50 \%$ of patients. Comorbidity risk score and the combinations of both heart and metabolic diseases, in particular, independently predict the effect of pulmonary rehabilitation on exercise capacity and quality of life.

In the context of the current evidence and taking the limitations into account, our findings enable physicians to carefully investigate the impact of comorbidities as potential predictors and confounders in the population of patients with COPD enrolled in rehabilitation programmes.

Acknowledgements: We gratefully acknowledge Daniela Lugli, BSc (Ospedale Villa Pineta) and Piera Ranieri, MD (Geriatric Research Group, Brescia, Italy), for their assistance in statistics and manuscript preparation. We also thank Roberto D'Amico, $\mathrm{PhD}$ (Service of Biostatistics, University of Modena-Reggio Emilia, Modena) for his helpful advice and revision of the statistical methods. We finally acknowledge Mary McKenney for editing the manuscript.

Competing interests: None.

Ethics approval: The study was approved by the institutional review board.

\section{REFERENCES}

1. World Health Report. Geneva: World Health Organization, 2000. http://www.who. int/whr/2000/en/ (accessed 17 March 2008).

2. Lopez AD, Shibuya K, Rao C, et al. Chronic obstructive pulmonary disease: current burden and future projections. Eur Respir J 2006;27:397-412.

3. Yach D, Hawkes C, Gould CL, et al. The global burden of chronic diseases. Overcoming impediments to prevention and control. JAMA 2004;291:2616-22.

4. Sevenoaks MJ, Stockley RA. Chronic obstructive pulmonary disease, inflammation and co-morbidity-a common inflammatory phenotype? Respir Res 2006; 7:70.

5. Van der Wel MC, Jansen RW, Bakx JC, et al. Non-cardiovascular co-morbidity in elderly patients with heart failure outnumbers cardiovascular co-morbidity. Eur $\mathrm{J}$ Heart Fail 2007; 9:709-15.

6. Guralnik JM, LaCroix AZ, Abbott RD, et al. Maintaining mobility in late life. I. Demographic characteristics and chronic conditions. Am J Epidemiol 1993;137:845-57.
7. Bellelli G, Guerini F, Bianchetti A, et al. Medical comorbidity and complexity of the rehabilitative procedures for older patients with functional impairments. J Am Geriatr Soc 2002:50:2095-6.

8. Kriegsman DM, Deeg DJ, Stalman WA. Comorbidity of somatic chronic diseases and decline in physical functioning; the Longitudinal Aging Study Amsterdam. J Clin Epidemiol 2004;57:55-65.

9. Soriano JB, Visick GT, Muellerova H, et al. Patterns of comorbidities in newly diagnosed COPD and asthma in primary care. Chest 2005;128:2099-107.

10. Sidney S, Sorel M, Quesenberry CP Jr, et al. COPD and incident cardiovascular disease hospitalizations and mortality: Kaiser Permanente Medical Care Program. Chest 2005;128:2068-75

11. Sin DD, Man SF. Chronic obstructive pulmonary disease as a risk factor for cardiovascular morbidity and mortality. Proc Am Thorac Soc 2005;2:8-11

12. Thun MJ, Henley SJ, Gansler T. Inflammation and cancer: an epidemiological perspective. Novartis Found Symp 2004;256:6-21.

13. Mannino DM, Watt G, Hole D, et al. The natural history of chronic obstructive pulmonary disease. Eur Respir J 2006;27:627-43.

14. Ferrer M, Alonso J, Morera J, et al. Chronic obstructive pulmonary disease stage and health-related quality of life in COPD patients. Respir Med 2001;95:496-504.

15. Wijnhoven HA, Kriegsman DM, Hesselink AE, et al. The influence of co-morbidity on health-related quality of life in asthma and COPD patients. Respir Med 2003;97:468-75

16. Van Manen J, Bindels P, Dekker F, et al. Added value of co-morbidity in predicting health-related quality of life in COPD patients. Respir Med 2001:95:496-504.

17. British Thoracic Society statement. Pulmonary rehabilitation. Thorax 2001; 56:827-34.

18. Roomi J, Jonson MM, Waters K, et al. Respiratory rehabilitation, exercise capacity and quality of life in chronic airways disease in old age. Age Ageing 1996;25:12-16.

19. Lacasse Y, Goldstein R, Lasserson TJ, et al. Pulmonary rehabilitation for chronic obstructive pulmonary disease. Cochrane Database Syst Rev 2006 18;(4):CD003793.

20. Griffiths TL, Phillips CJ, Davies S, et al. Cost effectiveness of an outpatient multidisciplinary pulmonary rehabilitation programme. Thorax 2001;56:779-84.

21. Global Initiative for Chronic Obstructive Pulmonary Disease. Global strategy for the diagnosis, management and prevention of chronic obstructive pulmonary disease: NHLBI/WHO workshop report, NIH Publication 2701. Bethesda, April 2001 (updated November 2006). http://www.goldcopd.com (accessed 11 March 2008).

22. International Classification of Diseases-9th revision. Office Edition Practice. http:// www.theodora.com/diseases/ (accessed 17 March 2008).

23. Classification Committee of WONCA. International Classification of Health Problems in Primary Care (ICHPPC)-2-defined. 3rd Edn. Oxford: Oxford University Press, 1983.

24. Charlson ME, Pompei P, Ales KL, et al. A new method of classifying prognostic comorbidity in longitudinal studies: development and validation. J Chronic Dis 1987:40:373-83.

25. Marti S, Munoz X, Rios J, et al. Body weight and comorbidity predict mortality in COPD patients treated with oxygen therapy. Eur Respir J 2006;27:689-96.

26. Romagnoli M, Dell'Orso D, Lorenzi $\mathbf{C}$, et al. Repeated pulmonary rehabilitation in severe and disabled COPD patients. Respiration 2006;73:769-76.

27. Rossi G, Florini F, Romagnoli $M$, et al. Length and effectiveness of pulmonary rehabilitation in outpatients with chronic airway obstruction. Chest 2005;127:105-9.

28. Redelmeier DA, Bayoumi AM, Goldstein RS, et al. Interpreting small differences in functional status: the Six Minute Walk test in chronic lung disease patients. Am J Respir Crit Care Med 1997;155:1278-82.

29. De Torres JP, Pinto-Plata V, Ingenito E, et al. Power of outcome measurements to detect clinically significant changes in pulmonary rehabilitation of patients with COPD. Chest 2002;121:1092-8.

30. Jones PW. Health status measurement in chronic obstructive pulmonary disease. Thorax 2001;56:880-7.

31. American Thoracic Society. Statement: Guidelines for the six-minute walk test. Am J Respir Crit Care Med 2002;166:111-17.

32. Fletcher CM. Standardised questionnaire on respiratory symptoms: a statement prepared and approved by the MRC Committee on the aetiology of chronic bronchitis (MRC breathlessness score). Br Med J 1960;2:1665.

33. Carone M, Bertolotti G, Anchisi F, et al. The St George's Respiratory Questionnaire (SGRO): Italian version. Rassegna Patol App Respir 1999;14:31-7.

34. Van Manen JG, Bindels PJE, ljzermans CJ, et al. Prevalence of comorbidity in patients with chronic airway obstruction and controls over the age of 40 . J Clin Epidemiol 2001;54:287-93.

35. Van Manen JG, Bindels PJE, Dekker FW, et al. The influence of COPD on healthrelated quality of life independent of the influence of comorbidity. J Clin Epidemiol 2003;56:1177-84.

36. Celli BR, Cote CG, Marin JM, et al. The Body-mass index, airflow obstruction, dyspnea, and exercise capacity index in chronic obstructive pulmonary disease. N Engl J Med 2004;350:1005-12.

37. Pinto-Plata V, Cote $\mathrm{C}$, Cabral $\mathrm{H}$, et al. The 6-min walk distance: change over time and value as a predictor of survival in severe COPD. Eur Respir J 2004;23:28-33.

38. De Fazio I, Franzoni S, Frisoni GB, et al. Predictive role of single diseases and their combination on recovery of balance and gait in disabled elderly patients. J Am Med Dir Assoc 2006;7:208-11.

39. Charlson M, Szatrowski TP, Peterson J, et al. Validation of a combined comorbidity index. J Clin Epidemiol 1994;47:1245-51.

40. Soyseth V, Brekke PH, Smith P, et al. Statin use is associated with reduced mortality in COPD. Eur Respir J 2007;29:279-83. 
41. Cote CG, Celli BR. Pulmonary rehabilitation and the BODE index in COPD. Eur Respir J 2005;26:630-6.

42. Expert panel on detection, evaluation, and treatment of high blood cholesterol in adults. JAMA 2001:285:2486-97.

43. Garcia-Aymerich $\mathbf{J}$, Lange $\mathrm{P}$, Benet $\mathrm{M}$, et al. Regular physical activity reduces hospital admission and mortality in chronic obstructive pulmonary disease: a population based cohort study. Thorax 2006:61:772-8.

44. Martinez FJ, Foster G, Curtis JL, et al, for the NETT Research Group. Predictors of mortality in patients with emphysema and severe airflow obstruction. Am J Respir Crit Care Med 2006;173:1326-34.

45. Troosters T, Casaburi R, Gosselink R, et al. Pulmonary rehabilitation in chronic obstructive pulmonary disease. Am J Respir Crit Care Med 2005;172:19-38.
46. World Health Organization. The world health report 2002: reducing risk, promoting healthy life. Geneva: World Health Organization, 2002.

47. Wang TJ, Evans JC, Benjamin EJ, et al. Natural history of asymptomatic left ventricular systolic dysfunction in the community. Circulation 2003:108:977-82.

48. European Society of Cardiology (Working Group Report). Recommendations for exercise training in chronic heart failure patients. Eur Heart $J$ 2001;22:125-35.

49. Lenzen M, Scholte op Reimer W, Norekval TM, et al. Pharmacological treatment and perceived health status during 1-year follow up in patients diagnosed with coronary artery disease, but ineligible for revascularization. Results from the Euro Heart Survey on Coronary Revascularization. Eur J Cardiovasc Nurs 2006;5:115-21.

50. Tinetti ME, Bogardus ST, Agostini JV. Potential pitfalls of disease-specific guidelines for patients with multiple conditions. N Engl J Med 2004;351:2870-4.

\section{Pulmonary puzzle}

\section{Progressive dyspnoea, pleural effusions and lytic bone lesions}

\section{CLINICAL PRESENTATION}

A 72-year-old man was referred following a CT scan which showed lytic and sclerotic lesions in the spine and pelvis with bilateral pleural effusions and thickening. The patient was an ex-smoker with progressive dyspnoea and significant weight loss over 1 year. There was no history of exposure to asbestos. Pleural fluid analysis revealed a transudate with no malignant cells. A repeat CT scan showed small bilateral pleural effusions and rinds of solid tissue within the pleural space extending to surround the descending thoracic aorta and aortic arch. Nodular reticular shadowing was seen in both lungs extending to the periphery, particularly in the upper lobes. Another soft tissue rind surrounded the kidneys with renal sinus fat obliteration. Presumed metastases were seen in both iliac bones with surrounding sclerosis on the left and the T7 vertebral body. An isotope bone scan revealed extensive focal increased tracer activity in lower limb long bones (fig 1). Initial pleural biopsy, performed to investigate the diagnosis of malignancy, showed non-specific fibrosis. Before further investigations could be undertaken, the patient died from aspiration pneumonia following a stroke. Because of the suspicion of mesothelioma, a coronial autopsy was undertaken which confirmed bilateral pleural thickening up to $1 \mathrm{~cm}$ with focal calcification but no macroscopic parenchymal infiltration.

\section{QUESTION}

What is the diagnosis?

See page 554

This case was submitted by:

\section{A G Nicholson, ${ }^{1}$ E Anderson, ${ }^{2}$ S Saha, ${ }^{3}$ M Indrajith, ${ }^{4}$ B Conry, ${ }^{5}$ J Hughes ${ }^{6}$}

${ }^{1}$ Department of Histopathology, Royal Brompton Hospital, London, UK; ${ }^{2}$ Department of Emergency Medicine, Queen Elizabeth Hospital NHS Trust, London, UK;

${ }^{3}$ Department of Ophthalmology, Guy's \& St Thomas' NHS Trust, London, UK;

${ }^{4}$ Department of Medicine, Royal Brompton Hospital, London, UK; ${ }^{5}$ Department of

Radiology, Maidstone \& Tunbridge Wells NHS Trust, Tunbridge Wells, Kent, UK

${ }^{6}$ Department of Medicine, Maidstone \& Tunbridge Wells NHS Trust, Tunbridge Wells, Kent, UK

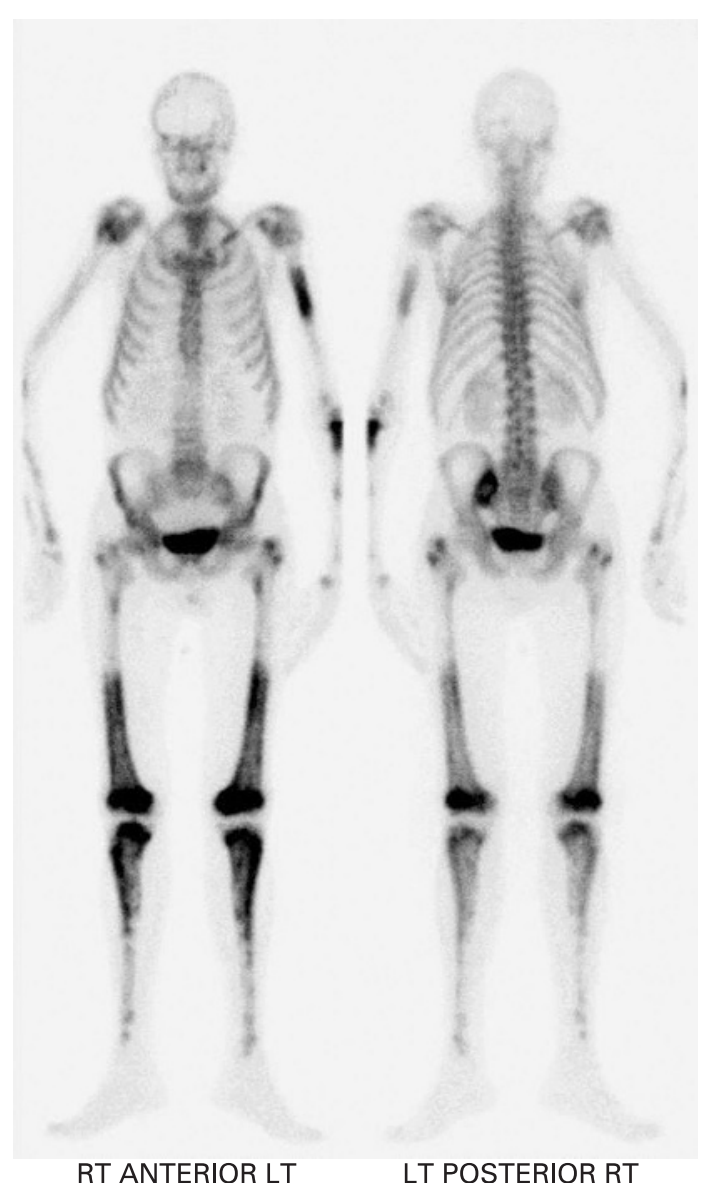

Correspondence to: Dr S Saha, Medical Eye Unit, Department of Ophthalmology, St Thomas' Hospital, London SE1 7EH, UK; shouvik.saha@doctors.org.uk

Competing interests: None.

Thorax 2008;63:492. doi:10.1136/thx.2007.091074 